\title{
Testing the potential of a ribosomal 165 marker for DNA metabarcoding of insects
}

Vasco Elbrecht, Pierre Taberlet, Tony Dejean, Alice Valentini, Philippe Usseglio-polatera, Jean-Nicolas Beisel, Eric Coissac, Frederic Boyer, Florian Leese

Cytochrome c oxidase I (COI) is a powerful marker for DNA barcoding of animals, with good taxonomic resolution and a large reference database. However, when used for DNA metabarcoding, estimation of taxa abundances and species detection are limited due to primer bias caused by highly variable primer binding sites across the $\mathrm{COI}$ gene. Therefore, we explored the ability of the $16 \mathrm{~S}$ ribosomal DNA gene as an alternative metabarcoding marker for species level assessments. Ten bulk samples, each containing equal amounts of tissue from 52 freshwater invertebrate taxa, were sequenced with the Illumina NextSeq 500 system. Both markers detected the same amount of taxa, but the $16 \mathrm{~S}$ primers amplified three more insect species and amplified more equally, probably due to decreased primer bias. Estimation of biomass might be less biased with $16 \mathrm{~S}$ than with COI, although variation in read abundances of two orders of magnitudes is still observed. According to these results, the marker choice depends on the scientific question. If the goal is to obtain a taxonomic identification at the species level, then COI is more appropriate due to established reference databases and known taxonomic resolution of this marker, knowing that a greater proportion of insects will be missed using COI Folmer primers. If the goal is to obtain a more comprehensive survey in a context where it is possible to build a local reference database, the $16 \mathrm{~S}$ marker or optimised degenerated COI primers could be more appropriate. 


\section{Testing the potential of a ribosomal $16 \mathrm{~S}$ marker for DNA}

\section{2 metabarcoding of insects}

3 Vasco Elbrecht ${ }^{1,2}$, Pierre Taberlet ${ }^{3,4}$, Tony Dejean ${ }^{5}$, Alice Valentini ${ }^{5}$, Philippe Usseglio-Polatera ${ }^{6}$, Jean-Nicolas

4 Beisel ${ }^{7,8}$, Eric Coissac ${ }^{3,4}$, Fred Boyer ${ }^{3,4}$, Florian Leese ${ }^{1,9}$

6 Affiliations:

7 1) Aquatic Ecosystem Research, Faculty of Biology, University of Duisburg-Essen, Universitätsstraße 5, 45141

8 Essen, Germany

9 2) Department of Animal Ecology, Evolution and Biodiversity, Ruhr University Bochum, Universitätsstraße 150,

1044801 Bochum, Germany

11 3) CNRS, Laboratoire d'Ecologie Alpine (LECA), 38000 Grenoble, France

12 4) Univ. Grenoble Alpes, Laboratoire d'Ecologie Alpine (LECA), 38000 Grenoble, France

13 5) SPYGEN, Savoie Technolac, 73370 Le Bourget du Lac, France

14 6) Univ Lorraine, CNRS, IBISE,UMR 7360, Lab Interdisciplinaire Environnements Continentaux, F-57070 Metz,

15 France

16 7) Ecole Natl Genie Eau \& Environm Strasbourg, F-67070 Strasbourg, France

17 8) Univ Strasbourg, UMR CNRS 7362, LIVE, F-67083 Strasbourg, France

18 9) Centre for Water and Environmental Research (ZWU) Essen, University of Duisburg-Essen, Universitätsstraße 2, 1945141 Essen,

Corresponding author: florian.leese@uni-due.de, $+49.201-1834053$, University of Duisburg-Essen, Faculty of Biology, Aquatic Ecosystem Research, Universitätsstr. 5, D-45141 Essen, Germany

\section{Abstract}

24 Cytochrome c oxidase I (COI) is a powerful marker for DNA barcoding of animals, with good taxonomic resolution and a large reference database. However, when used for DNA metabarcoding, estimation of taxa abundances and 
26 species detection are limited due to primer bias caused by highly variable primer binding sites across the COI gene.

27 Therefore, we explored the ability of the $16 \mathrm{~S}$ ribosomal DNA gene as an alternative metabarcoding marker for

28 species level assessments. Ten bulk samples, each containing equal amounts of tissue from 52 freshwater

29 invertebrate taxa, were sequenced with the Illumina NextSeq 500 system. Both markers detected the same amount of

30 taxa, but the $16 \mathrm{~S}$ primers amplified three more insect species and amplified more equally, probably due to decreased

31 primer bias. Estimation of biomass might be less biased with $16 \mathrm{~S}$ than with COI, although variation in read

32 abundances of two orders of magnitudes is still observed. According to these results, the marker choice depends on

33 the scientific question. If the goal is to obtain a taxonomic identification at the species level, then COI is more

34 appropriate due to established reference databases and known taxonomic resolution of this marker, knowing that a

35 greater proportion of insects will be missed using COI Folmer primers. If the goal is to obtain a more comprehensive

36 survey in a context where it is possible to build a local reference database, the 16S marker or optimised degenerated

37 COI primers could be more appropriate.

39 Keywords: Biodiversity assessment, stream monitoring, small ribosomal subunit, high throughput sequencing 


\section{Introduction}

41 DNA metabarcoding is a novel and powerful method to assess biodiversity in ecosystems (Hajibabaei et al., 2011;

42 Taberlet et al., 2012; Yu et al., 2012; Carew et al., 2013; Gibson et al., 2014; Leray \& Knowlton, 2015; Dowle et al., 2015). Well-designed universal PCR primers for the target group are the most critical component when assessing species diversity in ecosystems with DNA metabarcoding, because environmental samples typically contain hundreds of specimens of phylogenetically different taxa. Substantial primer bias in commonly used DNA barcoding markers, such as the Cytochrome c Oxidase subunit I (COI) gene for animals, prevents the detection of all taxa in a sample and thus the estimation of taxa biomass is difficult (Deagle et al., 2014; Piñol et al., 2014; Elbrecht \& Leese, 2015). However, accurate and comprehensive taxa lists are critical for assessment of biodiversity and ecosystem health. Given the great sequence variability of the COI marker, the use of alternative DNA metabarcoding markers has been suggested (Clarke et al., 2014, Deagle et al., 2014) and PCR-free metagenomics strategies are being tested for environmental assessment (Gómez-Rodríguez et al., 2015; Tang et al., 2015). One marker with potential for species level resolution and more conserved regions is the mitochondrial 16S rRNA gene (Clarke et al., 2014, Deagle et al., 2014). Clarke et al. 2014 has compared the performance of different COI and 16S primers on insect communities using an in silico approach which showed that the tested amplified ribosomal markers are generally more universal and detect more taxa than the COI markers. They also tested an insect mock sample containing DNA from 14 species with COI primers detecting or less taxa than with 16S. However, the performance of $16 \mathrm{~S}$ metabarcoding primers with aquatic invertebrate communities has not been extensively tested. In this study, we evaluate the performance of a short 16S region as compared to the standard COI Folmer (et al., 1994) marker for metabarcoding, using freshwater invertebrates mock communities. The ten freshwater mock communities were each comprised of 52 morphologically identified taxa and have been used in a previous study on $16 \mathrm{~S}$ fragment for DNA-based monitoring. Furthermore, this is a good test to see if our protocols developed for the MiSeq system can be easily ported to the NextSeq system without modifications, as the NextSeq and MiniSeq rely on sequencing by synthesis on only two instead of four channels for all four nucleotides like in older Illumina instruments (Illumina, Technical Note, 2016). 
66 Material and Methods

67 The same DNA aliquots as in Elbrecht \& Leese (2015) were used to test the 16S marker to allow for a direct comparison. Laboratory conditions and bioinformatic analyses were kept as similar as possible to the study by (Elbrecht \& Leese, 2015).

\section{DNA metabarcoding}

We used 16S markers ins_F / ins_R to amplify a $\sim 157 \mathrm{bp}$ of the mitochondrial $16 \mathrm{~S}$ gene. This marker was developed as part of this project using the ecoPrimers program (Riaz et al., 2011) and represents a variant of the Ins16S_1short primer pair (Clarke et al., 2014). Fusion primers were used (Figure S1), allowing to load PCR amplicons directly onto the Illumina NextSeq 500 sequencer. The same tag shifting and simultaneous sequencing of forward and reverse primer and 10\% PhiX spike in as described by (Lundberg et al., 2013; Elbrecht \& Leese, 2015) was used, to increase sequence diversity. Unique inline barcodes on forward and reverse reads were used for sample indexing.

The same one-step PCR and library preparation conditions as in (Elbrecht \& Leese, 2015) were used with the following modifications: PCR extension time was reduced to 120 seconds and annealing temperature increased to $52.5^{\circ} \mathrm{C}$ to better suit the fragment length and melting temperatures of the $16 \mathrm{~S}$ Ins primers. Only one PCR replicate per sample was used for sequencing. Amplicons were purified with magnetic beads, but only a left-sided size selection was carried out to remove remaining primers and primer dimers (0.9x SPRIselect, Beckman Coulter, Bread, CA, USA). Concentrations were quantified with the Qubit BR Kit (Thermofisher Scientific, Carlsbad, CA, USA) and the library for sequencing was prepared by pooling $190 \mathrm{ng}$ PCR product of all ten samples. cycles (150 bp PE sequencing) at the Alfred Wegener Institute Helmholtz Centre for Polar and Marine Research, Bremerhaven Germany.

\section{Generation of $16 \mathrm{~S}$ reference sequences}

91 Due to the limited availability of $16 \mathrm{~S}$ reference sequences on GenBank (NCBI), we constructed a reference library for the 52 morphotaxa used in this study, if tissue was still available. Standard DNA salt extraction, PCR, PCR 
93

94

clean-up, and Sanger sequencing were conducted as described in (Elbrecht et al., 2014), to amplify the 16S region with different primer sets and combinations. Primers were newly developed or checked for mismatches to Ephemeroptera, Plecoptera and Trichoptera using the PrimerMiner v0.2 R package (https://github.com/VascoElbrecht/PrimerMiner) and are available together with the generated reference sequences on BOLDsystems (DS-TMIX16S). An annealing temperature of $52^{\circ} \mathrm{C}$ was used for all primer combinations using HotMaster Taq (5Prime; Gaithersburg, Maryland, USA) for amplification.

\section{Bioinformatic analysis}

Figure S2B includes a flow chart of the data processing steps. All used custom R scripts are available in the supplementary information (S1 scripts). First, reads were demultiplexed (R script splitreads_ins_v11.R) and paired end reads merged using USEARCH v8.0.1623 -fastq_mergepairs with -fastq_merge_maxee 1.0 (Edgar \& Flyvbjerg, 2015). Primers were removed with cutadapt version 1.8.1 (Martin, 2011). Sequences from all ten replicates were pooled, dereplicated, and singletons were removed to find operational taxonomic units (OTUs) using the UPARSE pipeline (cluster_otus, 97\% identity, Edgar, 2013). Chimeras were removed from the OTUs using uchime_denovo. The remaining OTUs were identified by querying against all nucleotide records on NCBI using the Blast API (Entrez Programming Utilities) and an R script and our local 16S database using BLAST 2.2.31+ (Camacho et al., 2009). Taxonomy was assigned and checked manually, and in rare cases matches of $\sim 90 \%$ identity were accepted, if they matched the patterns which were previously reported for COI (Elbrecht \& Leese, 2015).

The ten samples were dereplicated using derep_fulllength, but singletons were included in the data set. Sequences of each sample were matched against the OTUs with a minimum match of $97 \%$ using usearch_global. The hit tables were imported and the sequence numbers were normalised to the total sequence abundance and tissue weight for the various taxa. Only OTUs with a read abundance above $0.003 \%$ in at least one replicate were considered in downstream analysis.

Due to the exponential nature of PCR, statistical tests on weight adjusted relative read abundances were carried out on decadic logarithm. Expected relative abundance was calculated by dividing $100 \%$ by the number of morphospecies detected with each marker. 


\section{Results}

\section{Amplicon sequencing success and sequence processing}

121 The NextSeq run generated $42.3 \mathrm{Gbp}$ of raw sequencing data (NCBI SRA accession number SRR2217415). Cluster

122

123

124

125

126

127

128

129

130

131

132

133

134

135 density was $177 \mathrm{~K} / \mathrm{mm}^{2}$ and read quality good with Q30 $\geq 85.3 \%$. Read abundance was 17\% higher when sequencing started with the P5_Ins_F primers (t-test, $\mathrm{p}<0.001$, Figure S2 A). This, however, did not introduce any significant differences between forward and reverse primer in the bioinformatic processing downstream ( $t$-test, Figure S2 B).

Initial OTU clustering generated 855 OTUs of which $22.5 \%$ were detected as chimeras. Sequences from each sample were compared against the remaining 663 OTUs, but only 243 OTUs had at least one sample with $>0.003 \%$ sequence abundance $(\sim 326$ reads, $\mathrm{SD}=29)$ and were thus included in further analysis. Taxonomy could be assigned for most OTUs based on available reference data and our own reference sequences. Reference data for the $16 \mathrm{~S}$ marker could be generated for 42 of the 52 morphotaxa by Sanger sequencing. Together with $16 \mathrm{~S}$ sequences from NCBI (Table S2) we were able to obtain reference data for all morphotaxa (Figure 1). However, in some cases the NCBI data was only obtained for taxa identified at family or order level (e.g. Lymnaeidae, Nematoda, Acari) and might not be sufficient for reliable taxa identification. Supplementary Table S1 gives an overview of assigned taxonomy for each OTU and table S2 shows the distribution of detected taxa across the 10 replicates. Table S3 shows the sequence abundance for each morphotaxon from the $16 \mathrm{~S}$ dataset as well as the COI dataset from (Elbrecht \& Leese, 2015), which was used for comparison of primer bias between both markers.

\section{Taxon recovery with $16 \mathrm{~S}$}

The taxonomic assignment was straigthforward for the COI marker, due to the availability of reliable reference databases, which was not the case for the $16 \mathrm{~S}$ marker. Forty-one out of 42 insect species were detected by the $16 \mathrm{~S}$. The Sanger sequence generated for the Tipulidae present in our mock samples showed 3 mismatches in 7 bases at the 3 ' end of both the forward and reverse $16 \mathrm{~S}$ primers and was not detected in the data set. 


\section{COI versus 16S}

143 Most insect taxa were amplified with both markers, (38 out of 42), no insect taxon was only detected by COI, while 144 16S detected three more taxa (Ephemeridae, Sercostoma personatum, Rhyacophyla). The 16S primers worked very 145 effectively for insect taxa, specifically in the indicator taxa Ephemeroptera, Plecoptera and Trichoptera (100\% 146 detection success, table 1). Of the ten other Metazoa, five were detected by COI, and only two by $16 \mathrm{~S}$. Variation in 147 logarithmic insect read abundance was much lower for the ribosomal $16 \mathrm{~S}$ amplicons $(\mathrm{SD}=0.62 \%)$ than for the COI 148 Folmer primers ( $\mathrm{SD}=1.0 \%)$ used on the same samples as in (Elbrecht \& Leese, 2015) (Fig 1). Logarithmic precision 149 of relative read abundance (distance to expected abundance) was significantly higher for $16 \mathrm{~S}(\mathrm{SD}=0.37)$ than for $150 \mathrm{COI}(\mathrm{SD}=0.72$, paired Wilcoxon signed-rank test, $\mathrm{p}=0.002)$. Additionally, the COI primers showed more dropouts 151 of a few specimens per taxa (orange numbers, figure 1), while the $16 \mathrm{~S}$ primer with the exception of three cases 152 always amplified all 10 specimens of a taxon. Table 1 compares the number of taxa recovered for the four most 153 relevant orders for water quality assessment. 


\section{Discussion}

We successfully ported our DNA metabarcoding protocol from the MiSeq system (Elbrecht \& Leese, 2015) onto the NextSeq 500 with good sequence quality and sequencing output as expected. As demonstrated in the previous study and also seen for the $16 \mathrm{~S}$ Ins marker here, the use of fusion primers with a parallel sequencing strategy maximizes sequence diversity (see Elbrecht \& Leese, 2015, Fig. S2), but can lead to a slight bias in read abundance. This however does not strongly affect read abundance of individual specimens between replicates (see Figure S5 \& S7 in Elbrecht \& Leese, 2015). As in the pervious test with COI Folmer primers, taxa not belonging to the 52 target taxa were detected with low abundances ( $<2 \%$ of data). This is likely a cause of trace DNA, gut content or small overlooked tissue pieces in the extraction, in some cases possibly ambiguous hits due to low identity to matches in the NCBI / BOLD databases. However, these reads are not posing an issue as they were excluded in the analysis (OTUs without hits, Fig. 1). Further, a slight bias on sequence abundance might be introduced between and within samples by e.g. different amount of cuticula present when weighing tissue, tissue quality and variation in mitochondrial copy number. However, these effects are the same for both markers, so observed effects can be likely explained by primer bias. Here, we focus on comparing the results obtained from sequencing the mock community of 52 taxa using the two different markers and discuss their advantages and disadvantages.

\section{Power and limitations of $16 \mathrm{~S}$ and COI markers in DNA metabarcoding}

A key advantage of COI as a marker for DNA metabarcoding is that reference databases have been well established and are actively developed and extended (Ratnasingham \& Hebert, 2007). DNA barcoding and the COI gene has been widely accepted by the scientific community as the barcoding marker of choice for animals (Ratnasingham \& Hebert, 2013; Porter et al., 2014), despite some negative voices (Taylor \& Harris, 2012). Additionally the taxonomic resolution of the COI marker has been extensively tested and its usefulness for identifying freshwater invertebrates on species level demonstrated (Zhou et al., 2009; Pfrender et al., 2010; Zhou et al., 2010; Sweeney et al., 2011). However, a documented concern of this marker is its large variability, which introduces primer bias due to mismatches at the primer binding sites (Piñol et al., 2014), which creates the risk of losing some target taxa (Clarke et al., 2014, Deagle et al., 2014). This large variation makes estimating biomass from PCR-based DNA metabarcoding results difficult (Elbrecht \& Leese, 2015). The results of this study show that the 16S Ins primers 
185

186

187

show less amplification bias than the COI Folmer primers previously tested, which is coherent with previous results from Clarke et al., 2014. Specifically for the Ephemeroptera, Plecoptera and Trichoptera, the 16S results were very consistent with variation in sequence abundance within these groups, with variation of only one order of magnitude magnitude for most taxa. A further advantage is that the reduced primer bias in $16 \mathrm{~S}$ could allow for lower sequencing depths and thus a reduction in costs. The downside of using $16 \mathrm{~S}$ as a marker at the present, however, is the limited availability of reference sequences and the yet not fully explored taxonomic resolution on species level. We had to establish our own $16 \mathrm{~S}$ reference sequences for our mock communities de novo whenever tissue of the analysed morphotaxa was still available. This created extra work and cost that was omitted when using COI.

\section{Which marker to use?}

$\mathrm{COI}$ is the standard marker for barcoding of animals and will typically yield the best resolved taxonomic lists.

Therefore, if the goal of a project is to obtain a taxonomic identification at the species level, COI is most appropriate. However due to the codon degeneracy some taxa will likely not be amplified and thus missing in the dataset, making the COI marker not ideal when complete taxon lists are required. In direct comparison, three more insect taxa were not detected with the COI Folmer primers but found with the 16S Ins primers. Also Clarke et al., (2014) showed for various COI primers that they performed either equally well or worse than the tested 16S primers. However, the use of improved COI primers with high degeneracy might lead to equally good detection and amplification consistency and would allow us to take advantage of existing reference databases. Degenerated COI primers like the "mlCOIint" primer sets by Leray et al., (2013) are being used in metabarcoding studies, but evaluation concerning their primer bias is needed. Both the COI and $16 \mathrm{~S}$ primer showed problems amplifying taxa listed in "other metazoa". This, however, is expected as the Ins primers are optimised for insect taxa and the Folmer primers were developed in the early 1990s with very little reference data available. Additionally, many morphotaxa are phylogenetically distant from insects, making them more difficult to target with universal primers. If the project goal is to obtain a more comprehensive survey and where it is possible to build a local reference database $16 \mathrm{~S}$ can be a versatile and possibly even better alternative to COI, as this marker minimizes primer bias and provides more consistent PCR. Thus, $16 \mathrm{~S}$ may possibly allow for rough biomass inferences, yet the variation of still two orders of magnitude as shown in this study show clear limitations as well. For species-level assignments, the potential of $16 \mathrm{~S}$ remains largely unexplored for assessment of relevant invertebrate indicator taxa such as 
212 Ephemeroptera, Plecoptera and Trichoptera. Thus, prior to a routine application on 16S for species-level assessment

213 we recommend reference sequencing of whole mitochondrial genomes using high throughput sequencing (Tang et

214 al., 2014), which not only allows for estimating taxonomic resolution of the two different mitochondrial markers,

215 but also build the backbone for future metagenomic studies (Tang et al., 2015). Once comprehensive mitochondrial

216 reference databases are established, also direct PCR-free shotgun sequencing of bulk samples (metagenomics)

217 becomes feasible. These approaches could further improve taxa detection rates and potentially allow to estimate taxa 218 abundance (Gómez-Rodríguez et al., 2015; Tang et al., 2015). Using methods to enrich for mitochondrial reads we

219 could further decrease sequencing costs for reference sequencing and mitogenomics approaches alike (Zhou et al., 220 2013; Liu et al., 2015; Dowle et al., 2015).

\section{Conclusions}

In this study we show that the ribosomal $16 \mathrm{~S}$ marker shows less primer bias than the COI barcoding marker with Folmer primers, when applied for DNA metabarcoding of freshwater insects. Thus the developed 16S primers might allow to reduce sequencing depth in DNA based stream assessment, which could reduce sequencing costs. The main drawback when compared to $\mathrm{COI}$ is that little reference databases for stream invertebrates are available for the $16 \mathrm{~S}$ marker and that taxonomic resolution remains largely unknown. This might change in the future when more reference data is generated, especially in the form of complete mitochondrial genomes generated with high throughput sequencing approaches. In cases where it is viable to generate local reference databases $16 \mathrm{~S}$ could be a suitable alternative to COI. Additionally, degenerated COI primers should be evaluated as the are likely to perform better than the herein studied Folmer primers, which are not optimised for metabarcoding. 


\section{Acknowledgements:}

234 We thank Uwe John and Nancy Kuehne (Alfred Wegener Institute Helmholtz Centre for Marine and Polar 235 Research, Bermerhaven) for running the library on the MiSeq sequencer. We thank Laurence Clarke, 236 Nathan Bott and one anonymous reviewer for helpful comments that substantially improved this study. 237

238 Declaration of interest: Tony Dejean and Alice Valentini are employees of SPYGEN, Savoie Technolac.

239 The other authors declare that they have no competing interests. 


\section{Figures}

Figure 1: Comparison of 16S Ins (A) and COI Folmer (B) primer performance, both tested with the same 10 bulk samples each containing 52 morphologically distinct macroinvertebrate taxa. The 52 taxa are shown on the $\mathrm{x}$-axis with the number of reads obtained with $16 \mathrm{~S}$ and $\mathrm{COI}$ for each sample indicated by black dots on the logarithmic $\mathrm{y}-$ axis (mean relative abundance of detected morphotaxa is indicated by red circles). Sequence abundance was normalized across the ten replicates and the amount of tissue used in each DNA extraction. Only OTUs which had minimum abundance of $0.003 \%$ in at least one of the 10 samples were included in the analysis. Number of samples for which a morphotaxon was not detected is indicated by orange and red numbers in each plot. A thick vertical line in light red indicates if a morphotaxon was not detected. Detection rates between $16 \mathrm{~S}$ and COI marker are summarized in a Venn diagram. The availability of $16 \mathrm{~S}$ reference data from NCBI and own Sanger sequences is indicated by yellow and green background colour behind the taxon names on the x-axis. 


\section{Supplementary information}

254 Figure S1: 16S fusion primers used in this study

255 Figure S2: Distribution of reads obtained by NextSeq and number of reads discarded throughout the different 256 bioinformatics processing steps.

258 Table S1: Sequence of each OTU with abundance of assigned reads and assigned taxonomy.

259 Table S2: Distribution of OTUs across the 52 taxa.

260 Table S3: Raw number of reads assigned to each of the 52 taxa for $16 \mathrm{~S}$ and COI across the 10 replicates.

262 Scripts S1: R scripts used in this study to process sequence data and create plots 
Camacho C, Coulouris G, Avagyan V, Ma N, Papadopoulos J, Bealer K, Madden TL 2009. BLAST+: architecture and applications. BMC bioinformatics 10:421.

Carew ME, Pettigrove VJ, Metzeling L, Hoffmann AA 2013. Environmental monitoring using next generation sequencing: rapid identification of macroinvertebrate bioindicator species. Frontiers in zoology 10:1-1.

Clarke LJ, Soubrier J, Weyrich LS, Cooper A 2014. Environmental metabarcodes for insects: in silicoPCR reveals potential for taxonomic bias. Molecular ecology resources 14:1160-1170.

Deagle BE, Jarman SN, Coissac E, Pompanon F, Taberlet P 2014. DNA metabarcoding and the cytochrome c oxidase subunit I marker: not a perfect match. Biology Letters 10:20140562-20140562.

Dowle EJ, Pochon X, Banks J, Shearer K, Wood SA 2015. Targeted gene enrichment and high throughput sequencing for environmental biomonitoring: a case study using freshwater macroinvertebrates. Molecular ecology resources: $\mathrm{n} / \mathrm{a}-\mathrm{n} / \mathrm{a}$.

Edgar RC 2013. UPARSE: highly accurate OTU sequences from microbial amplicon reads. Nature Methods 10:996-998.

Edgar RC, Flyvbjerg H 2015. Error filtering, pair assembly and error correction for next-generation sequencing reads. Bioinformatics 31:3476-3482.

Elbrecht V, Leese F 2015. Can DNA-Based Ecosystem Assessments Quantify Species Abundance? Testing Primer Bias and Biomass-Sequence Relationships with an Innovative Metabarcoding Protocol. PloS one 10:e0130324-16.

Elbrecht V, Feld CK, Gies M, Hering D, Sondermann M 2014. Genetic diversity and dispersal potential of the stonefly Dinocras cephalotes in a central European low mountain range. Freshwater Science 33:181-192.

Folmer O, Black M, Hoeh W, Lutz R, Vrijenhoek R 1994. DNA primers for amplification of mitochondrial cytochrome $\mathrm{c}$ oxidase subunit I from diverse metazoan invertebrates. Molecular marine biology and biotechnology 3:294-299.

Gibson J, Shokralla S, Porter TM, King I, van Konynenburg S, Janzen DH, Hallwachs W, Hajibabaei M 2014. Simultaneous assessment of the macrobiome and microbiome in a bulk sample of tropical arthropods through DNA metasystematics. Proceedings of the National Academy of Sciences 111:8007-8012.

Gómez-Rodríguez C, Crampton-Platt A, Timmermans MJTN, Baselga A, Vogler AP 2015. Validating the power of mitochondrial metagenomics for community ecology and phylogenetics of complex assemblages. Methods in Ecology and Evolution 6:883-894.

Hajibabaei M, Shokralla S, Zhou X, Singer G, Baird DJ 2011. Environmental Barcoding: A Next-Generation Sequencing Approach for Biomonitoring Applications Using River Benthos. PloS one.

Illumina, Technical Note. (2016) Illumina Two-Channel SBS Sequencing Technology. Pub. No. 770-2013-054 Current as of 04 January 2016.

Leray M, Yang JY, Meyer CP, Mills SC, Agudelo N, Ranwez V, Boehm JT, Machida RJ 2013. A new versatile primer set targeting a short fragment of the mitochondrial COI region for metabarcoding metazoan diversity: application for characterizing coral reef fish gut contents. Frontiers in zoology 10:1-1.

Leray M, Knowlton N 2015. DNA barcoding and metabarcoding of standardized samples reveal patterns of marine benthic diversity. Proceedings of the National Academy of Sciences of the United States of America:201424997-6.

Liu S, Wang X, Xie L, Tan M, Li Z, Su X, Zhang H, Misof B, Kjer KM, Tang M, Niehuis O, Jiang H, Zhou X 2015. Mitochondrial capture enriches mito-DNA 100 fold, enabling PCR-free mitogenomics biodiversity analysis. Molecular ecology resources.

Lundberg DS, Yourstone S, Mieczkowski P, Jones CD, Dangl JL 2013. Practical innovations for high-throughput amplicon sequencing. Nature Methods 10:999-1002.

Martin M 2011. Cutadapt removes adapter sequences from high-throughput sequencing reads. EMBnet journal $17: 10-12$.

Pfrender ME, Hawkins CP, Bagley M, Courtney GW, Creutzburg BR, Epler JH, Fend S, Schindel D, Ferrington LC, Hartzell PL, Jackson S, Larsen DP, Lévesque A, Morse JC, Petersen MJ, Ruiter D, Whiting M 2010. Assessing macroinvertebrate biodiversity in freshwater ecosystems: advances and challenges in DNA-based approaches. The Quarterly review of biology 85:319-340.

Piñol J, Mir G, Gomez-Polo P, Agustí N 2014. Universal and blocking primer mismatches limit the use of highthroughput DNA sequencing for the quantitative metabarcoding of arthropods. Molecular ecology resources:1- 
12.

Porter TM, Gibson JF, Shokralla S, Baird DJ, Golding GB, Hajibabaei M 2014. Rapid and accurate taxonomic classification of insect (class Insecta) cytochrome coxidase subunit 1 (COI) DNA barcode sequences using a naïve Bayesian classifier. Molecular ecology resources 14:n/a-n/a.

Ratnasingham S, Hebert P 2007. BOLD: The Barcode of Life Data System (http://www. barcodinglife. org). Molecular Ecology Notes 7:355-364.

Ratnasingham S, Hebert PDN 2013. A DNA-Based Registry for All Animal Species: The Barcode Index Number (BIN) System. PloS one 8:e66213-16.

Riaz T, Shehzad W, Viari A, Pompanon F, Taberlet P, Coissac E 2011. ecoPrimers: inference of new DNA barcode markers from whole genome sequence analysis. Nucleic acids research 39:e145-e145.

Sweeney BW, Battle JM, Jackson JK, Dapkey T 2011. Can DNA barcodes of stream macroinvertebrates improve descriptions of community structure and water quality? Journal of the North American Benthological Society 30:195-216.

Taberlet P, Coissac E, Hajibabaei M, Rieseberg LH 2012. Environmental DNA. Molecular Ecology 21:1789-1793. Tang M, Hardman CJ, Ji Y, Meng G, Liu S, Tan M, Yang S, Moss ED, Wang J, Yang C, Bruce C, Nevard T, Potts SG, Zhou X, Yu DW 2015. High-throughput monitoring of wild bee diversity and abundance via mitogenomics. Methods in Ecology and Evolution 6:1034-1043.

Tang M, Tan M, Meng G, Yang S, Su X, Liu S, Song W, Li Y, Wu Q, Zhang A, Zhou X 2014. Multiplex sequencing of pooled mitochondrial genomes-a crucial step toward biodiversity analysis using mitometagenomics. Nucleic acids research 42:gku917-e166.

Taylor HR, Harris WE 2012. An emergent science on the brink of irrelevance: a review of the past 8 years of DNA barcoding. Molecular ecology resources:no-no.

Yu DW, Ji Y, Emerson BC, Wang X, Ye C, Yang C, Ding Z 2012. Biodiversity soup: metabarcoding of arthropods for rapid biodiversity assessment and biomonitoring. Methods in Ecology and Evolution 3:613-623.

Zhou X, Adamowicz SJ, Jacobus LM, DeWalt RE, Hebert PD 2009. Towards a comprehensive barcode library for arctic life - Ephemeroptera, Plecoptera, and Trichoptera of Churchill, Manitoba, Canada. Frontiers in zoology $6: 30$.

Zhou X, Jacobus LM, DeWalt RE, Adamowicz SJ, Hebert PDN 2010. Ephemeroptera, Plecoptera, and Trichoptera fauna of Churchill (Manitoba, Canada): insights into biodiversity patterns from DNA barcoding. Journal of the North American Benthological Society 29:814-837.

Zhou X, Li Y, Liu S, Yang Q, Su X, Zhou L, Tang M, Fu R, Li J, Huang Q 2013. Ultra-deep sequencing enables high-fidelity recovery of biodiversity for bulk arthropod samples without PCR amplification. GigaScience 2:4. 


\section{Figure 1 (on next page)}

Comparison of $16 \mathrm{~S}$ Ins (A) and COI Folmer (B) primer performance, both tested with the same 10 bulk samples each containing 52 morphologically distinct macroinvertebrate taxa.

Figure 1: Comparison of $16 \mathrm{~S}$ Ins (A) and COI Folmer (B) primer performance, both tested with the same 10 bulk samples each containing 52 morphologically distinct macroinvertebrate taxa. The 52 taxa are shown on the $x$-axis with the number of reads obtained with $16 \mathrm{~S}$ and $\mathrm{COI}$ for each sample indicated by black dots on the logarithmic $\mathrm{y}$-axis (mean relative abundance of detected morphotaxa is indicated by red circles). Sequence abundance was normalized across the ten replicates and the amount of tissue used in each DNA extraction. Only OTUs which had minimum abundance of $0.003 \%$ in at least one of the 10 samples were included in the analysis. Number of samples for which a morphotaxon was not detected is indicated by orange and red numbers in each plot. A thick vertical line in light red indicates if a morphotaxon was not detected. Detection rates between $16 \mathrm{~S}$ and $\mathrm{COI}$ marker are summarized in a Venn diagram. The availability of $16 \mathrm{~S}$ reference data from NCBI and own Sanger sequences is indicated by yellow and green background colour behind the taxon names on the $x$-axis. 

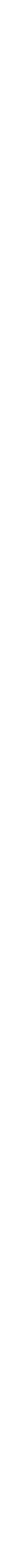


\section{Table $\mathbf{1}$ (on next page)}

Table 1. Number of specimens recovered with the COI and $16 \mathrm{~S}$ primers.

Table 1 .[b] Number of specimens recovered with the $\mathrm{COI}$ and $16 \mathrm{~S}$ primers. 
1 Table 1. Number of specimens recovered with the COI and 16S primers.

\begin{tabular}{|c|c|c|c|c|}
\hline \multirow[t]{2}{*}{ Taxonomic group } & \multicolumn{4}{|c|}{ Recovered specimens } \\
\hline & & $\mathrm{COI}$ & & $16 \mathrm{~S}$ \\
\hline Ephemeroptera & $7 / 8$ & $(88 \%)$ & $8 / 8$ & $(100 \%)$ \\
\hline Plecoptera & $4 / 4$ & $(100 \%)$ & $4 / 4$ & $(100 \%)$ \\
\hline Trichoptera & $13 / 15$ & $(86 \%)$ & $15 / 15$ & $(100 \%)$ \\
\hline Diptera & $7 / 8$ & $(88 \%)$ & $7 / 8$ & $(88 \%)$ \\
\hline Other insects & $7 / 7$ & $(100 \%)$ & $7 / 7$ & $(100 \%)$ \\
\hline Other metazoa & $5 / 10$ & $(50 \%)$ & $2 / 10$ & $(20 \%)$ \\
\hline$\Sigma$ All insects & $38 / 42$ & $(91 \%)$ & $41 / 42$ & $(98 \%)$ \\
\hline$\Sigma$ All taxa & $43 / 52$ & $(83 \%)$ & $43 / 52$ & $(83 \%)$ \\
\hline
\end{tabular}

